\title{
Including Future Consumption and Production in Economic Evaluation of Interventions that Save Life-Years: Commentary
}

\author{
Erik Nord $^{1}$ (1) $\cdot$ Christoffer Lamøy ${ }^{2}$
}

Published online: 30 April 2018

(C) The Author(s) 2018

In standard economic evaluation of competing health programmes, it is not customary to account for consumption and production in life-years gained. Whether or not these items ought to be included on the cost side has been the subject of much theoretical debate [1-12].

The purpose of this commentary is not to pick up all the loose ends of that debate but to drive home an inescapable analytical point: All else equal, a given number of qualityadjusted life-years (QALYs) from life prolongation will normally be more costly from a societal perspective than the same number of QALYs from programmes that improve quality of life. It is a matter for debate how much this fact should affect society's priority setting between quality-improving and life-prolonging programmes. But this normative issue should not bar the inclusion of consumption and production effects in economic analyses when their purpose is to show decision makers the societal opportunity costs of different courses of action.

Consider two ways of spending a million euros on healthcare in a community. In alternative A, ten people will each get an improvement in quality of life of 0.1 on the $0-1$ utility scale used in QALY calculations, and each will get to enjoy this for 10 years. The gain (undiscounted) is 10 QALYs. In alternative B, one person will gain 10 years of life in perfect health. Again, the undiscounted gain is 10 QALYs. Given that the intervention costs are the same, the two options at first glance seem to be on a par economically

Erik Nord

erik.nord@fhi.no

1 Norwegian Institute of Public Health, P.O. Box 4404, Nydalen, 0403 Oslo, Norway

2 School of Pharmacy, University of Oslo, P.O. Box 1068, Blindern, 0316 Oslo, Norway speaking, but, in fact, they are not. In alternative B, for the benefit of 10 QALYs to be realized, the intervention itself must be followed up by provision of means to the saved person so that he/she can actually live the saved years. The costs of these means are value (consumption) sacrificed by the rest of society. In alternative A, there are no such additional costs to the rest of society, since the years lived by the ten individuals who get increased quality of life are years that would have been lived anyway.

The sacrifice made by the rest of society to sustain the person whose life is prolonged may be offset by production by that person in the saved years. A 'net sustainment cost' may then be calculated. Sometimes this will be a negative number or, in other words, a net gain. But, in a wide range of life-prolonging programmes, saved life-years will typically have positive net sustainment costs because most saved life-years are provided to elderly people with low or no work contribution to society.

Regarding the consumption component, there is already consensus among health economists that necessary medical follow-up treatment for the condition in question (so-called related medical costs) needs to be part of the cost assessment [13]. Beyond that, willingness among health economists to accept 'medical treatments for other conditions' ('unrelated medical costs') as relevant in cost assessment has been greater than acceptance of other types of private or public consumption. But our question is simply how many resources are taken from other possible uses as a consequence of patients living longer and consuming while they do so. There is then no reason to distinguish between different kinds of consumption.

It follows from this reasoning that the 10 QALYs gained in alternative $\mathrm{B}$ are statistically more costly to society than those in alternative A. This translates into a general point: All else equal, a given number of QALYs from life 
prolongation will normally be more costly from a societal perspective than the same number of QALYs from programmes that improve quality of life. The same applies if one uses a measure of benefit other than the QALY (e.g. 'equity-weighted QALYs') or another type of economic analysis [14]. If two benefits with equal direct intervention costs are considered to have the same value from societal decision makers' point of view, and one comes from improving quality of life and the other comes from prolonging life, then the former benefit will be less costly to achieve than the latter.

As noted in the introduction, it is not customary to take this into account in standard economic evaluation of competing health programmes. A reason often given is that if consumption and production gains in life-years gained were to be included, and cost-effectiveness ratios were emphasised in priority setting across programmes, the effect would be to disadvantage elderly people relative to young people, since net sustainment costs tend to be much higher in late years of life than in early years of life. To disadvantage elderly people in need of life-prolonging interventions by holding against them their sustainment costs in terms of pensions seems quite problematic, both ethically and politically.

Our position is that a fair and obvious task in health economics is to make clear what different interventions actually cost society. A different question is how much the estimated costs should count in priority setting between interventions for different patient groups. We believe that if these two questions are separated-one factual and the other normative-there should be little ground for disagreement regarding the legitimacy and usefulness of answering the factual question.

As part of a master's thesis, one author (CL) recently examined 20 earlier publications on cost effectiveness in life-prolonging interventions. These publications covered 14 different conditions and 29 different interventions with medication that reduced mortality risk. Costs and effects were evaluated in a total of 56 different age and sex subgroups $[15,16]$. We added estimates of consumption costs and production gains to the direct intervention costs that were already covered. On average, the costs per life-year gained were almost tripled when consumption costs were included and almost doubled when production gains were also taken into account.

Our estimates pertain to fairly old studies and are thus of limited value. Further analyses using more recent studies are needed. Irrespective of the exact outcome of such research, we suggest that societal decision makers responsible for resource allocation in healthcare should consider placing some weight on consumption costs and production gains when prioritising between interventions for different patient groups. How much weight, and how this might be formalized in economic evaluation of healthcare, are-in our view-issues for further exploration by both researchers and policy makers.

\section{Compliance with Ethical Standards}

Funding No sources of funding were used to conduct this study or prepare this manuscript.

Conflict of interest Erik Nord and Christopher Lamøy have no conflicts of interest directly relevant to the content of this commentary.

Open Access This article is distributed under the terms of the Creative Commons Attribution-NonCommercial 4.0 International License (http://creativecommons.org/licenses/by-nc/4.0/), which permits any noncommercial use, distribution, and reproduction in any medium, provided you give appropriate credit to the original author(s) and the source, provide a link to the Creative Commons license, and indicate if changes were made.

\section{References}

1. Meltzer D. Accounting for future costs in medical cost-effectiveness analysis. J Health Econ. 1997;16:33-64.

2. Nyman JA. Should the consumption of survivors be included as a cost in cost-utility analysis? Health Econ. 2004;13:417-27.

3. Richardson JR, Olsen JA. In defence of societal sovereignty: a comment on Nyman 'the inclusion of survivor consumption in CUA'. Health Econ. 2006;15:311-3.

4. Lundin D, Ramsberg J. On survival consumption costs-a reply to Nyman. Health Econ. 2008;17:293-7.

5. Gandjour A. Consumption costs and earnings during added years of life-a reply to Nyman. Health Econ. 2006;15:315-7.

6. Nyman JA. More on survival consumption costs in cost-utility analysis. Health Econ. 2006;15:319-22.

7. Lee RH. Future costs in cost effectiveness analysis. J Health Econ. 2008;27:809-18.

8. Feenstra TL, et al. Future costs in economic evaluation: a comment on Lee. J Health Econ. 2008;27:645-1649.

9. Johannesson M, Meltzer D, O'Conor RM. Incorporating future costs in medical cost-effectiveness analysis: implications for the cost-effectiveness of the treatment of hypertension. Med Decis Mak. 1997;17:382-9.

10. Meltzer D, et al. Effect of future costs on cost-effectiveness of medical interventions among young adults: the example of intensive therapy for type 1 diabetes mellitus. Med Care. 2000;38:679-85.

11. Manns B, et al. Illustrating the impact of including future costs in economic evaluations: an application to end-stage renal disease care. Health Econ. 2003;12:949-58.

12. Norwegian Ministry of Health. White Paper on Priority Setting. Oslo; 2016.

13. Gros B, Soto Álvarez J, Casado MA. Incorporation of future costs in health economic analysis publications: current situation and recommendations for the future. Exp Rev Pharmacoecon Outcomes Res. 2015;15:465-9.

14. Nord E. Beyond QALYs: multi-criteria based estimation of maximum willingness to pay for health technologies. Eur J Health Econ. 2017. https://doi.org/10.1007/s10198-017-0882-x.

15. Tengs TO, et al. Five-hundred life-saving interventions and their cost-effectiveness. Risk Anal. 1995;15:369-90.

16. Lamøy C. Including consumption costs and production gains when pharmaceutical yield more life years. Master thesis. Oslo: University of Oslo; 2017. 